\title{
p62/SQSTM1-induced caspase-8 aggresomes are essential for ionizing radiation-mediated apoptosis
}

\author{
Su Hyun Lee $\mathbb{D}^{1,2,9}$, Won Jin Cho ${ }^{1,9}$, Abdo J. Najy ${ }^{1}$, Allen-Dexter Saliganan ${ }^{1}$, Tri Pham ${ }^{1}$, Joseph Rakowski ${ }^{3,4}$, Brian Loughery ${ }^{3}$, \\ Chang Hoon $\mathrm{Ji}^{2,5}$, Wael Sakr ${ }^{1}$, Seongho Kim ${ }^{3}$, Ikuko Kato ${ }^{3}$, Weon Kuu Chung ${ }^{6}$, Harold E. Kim ${ }^{3,4}$, Yong Tae Kwon (D ${ }^{2,5,7,8 凶}$ and \\ Hyeong-Reh C. Kim (iD) ${ }^{1,3 凶}$
}

(c) The Author(s) 2021

The autophagy-lysosome pathway and apoptosis constitute vital determinants of cell fate and engage in a complex interplay in both physiological and pathological conditions. Central to this interplay is the archetypal autophagic cargo adaptor p62/SQSTM1/ Sequestosome-1 which mediates both cell survival and endoplasmic reticulum stress-induced apoptosis via aggregation of ubiquitinated caspase-8. Here, we investigated the role of p62-mediated apoptosis in head and neck squamous cell carcinoma (HNSCC), which can be divided into two groups based on human papillomavirus (HPV) infection status. We show that increased autophagic flux and defective apoptosis are associated with radioresistance in HPV(-) HNSCC, whereas HPV(+) HNSCC fail to induce autophagic flux and readily undergo apoptotic cell death upon radiation treatments. The degree of radioresistance and tumor progression of HPV(-) HNSCC respectively correlated with autophagic activity and cytosolic levels of p62. Pharmacological activation of the p62-ZZ domain using small molecule ligands sensitized radioresistant HPV(-) HNSCC cells to ionizing radiation by facilitating p62 self-polymerization and sequestration of cargoes leading to apoptosis. The self-polymerizing activity of p62 was identified as the essential mechanism by which ubiquitinated caspase- 8 is sequestered into aggresome-like structures, without which irradiation fails to induce apoptosis in HNSCC. Our results suggest that harnessing p62-dependent sequestration of ubiquitinated caspase-8 provides a novel therapeutic avenue in patients with radioresistant tumors.

Cell Death and Disease (2021)12:997; https://doi.org/10.1038/s41419-021-04301-7

\section{INTRODUCTION}

Crosstalk and interplay among programmed cell death and cell survival pathways play a critical role in normal cellular processes as well as under pathological conditions [1-4]. While apoptosis is most responsible for programmed cell death induced by developmental cues, intracellular damage or external death stimuli, autophagy is a major cell survival pathway that removes damaged cellular components and supports cellular metabolism $[5,6]$. Autophagy has dual effects on cancer development and progression. It exerts tumor suppressive functions by removing damaged organelles and pathogens, contributing to the maintenance of genomic stability in normal cells [7]. However, as cancers progress, autophagy promotes cancer cell survival, thereby exerting oncogenic activity for cancer progression and therapy-resistance $[8,9]$. Given that the impairment of the delicate interplay between autophagy and apoptosis is a contributing factor in the pathogenesis of many human diseases including cancers, efforts have been made to find the molecular and functional points of interactions between autophagy and apoptosis [10, 11]. In preclinical studies, pharmacological inhibition or genetic manipulation of autophagic regulators such as autophagy-related proteins (ATGs), mammalian target of rapamycin (mTOR), and phosphatidylinositol 3-kinase catalytic subunit type 3 (PI3KC3) have been shown to sensitize tumor cells to chemotherapy-induced cell death $[9,12,13]$. These studies have led to multiple clinical trials using autophagy inhibitors such as chloroquine or hydroxychloroquine as a strategy to promote cell death. Although this approach showed minor improvements in clinical trials, there are concerns about specificity and side effects associated with the systemic inhibition of autophagy [13, 14]. Thus, a critical need persists for the development of diseasespecific and mechanism-based therapeutic tools to manipulate autophagic cell survival and programmed cell death.

The $\mathrm{N}$-degron pathway is a proteolytic system wherein single $\mathrm{N}$-terminal amino acids of proteins function as degradation determinants, called $\mathrm{N}$-degrons, which are recognized by the recognition components (N-recognins) $[15,16]$. The $\mathrm{N}$-degrons include Arg, Lys, His (type 1; positively charged), Phe, Tyr, Trp, Leu,

\footnotetext{
${ }^{1}$ Department of Pathology, Wayne State University School of Medicine, Karmanos Cancer Institute, Detroit, MI 48201, USA. ${ }^{2}$ Cellular Degradation Biology Research Center and

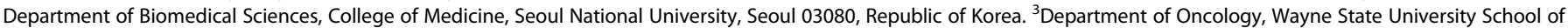

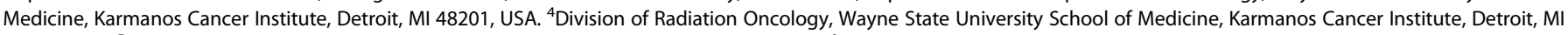
48201, USA. ${ }^{5}$ AUTOTAC Bio Inc., Changkkyunggung-ro 254, Jongno-gu, Seoul 03080, Korea. ${ }^{6}$ Department of Radiation Oncology, Kyung Hee University Hospital at Gangdong, College of Medicine, Kyung Hee University, Seoul, Korea. ${ }^{7}$ SNU Dementia Research Center, College of Medicine, Seoul National University, Seoul 03080, Republic of Korea.

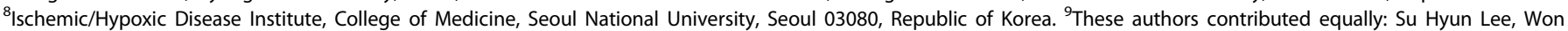
Jin Cho. ${ }^{凶}$ email: yok5@snu.ac.kr; hrckim@med.wayne.edu

Edited by Professor Gian Maria Fimia
}

Received: 8 February 2021 Revised: 3 September 2021 Accepted: 7 October 2021

Published online: 25 October 2021 
and lle (type 2; bulky hydrophobic), as well as ATE1-mediated arginylation-permissive residues, exposed at the protein termini $[17,18]$. The human genome encodes a set of $\mathrm{N}$-recognins (UBR1, UBR2, UBR4, and UBR5) whose UBR-box domains bind N-degrons for substrate ubiquitination and proteasomal degradation [19, 20]. Recently, we found that the N-degron pathway also mediates autophagic proteolysis where the autophagic receptor p62/ SQSTM1 acts as an N-recognin that binds N-degrons [21, 22]. In this process, the Nt-Arg residue of $\mathrm{N}$-degrons binds the p62 ZZ domain to induce p62 self-polymerization and interaction with LC3 on autophagic membranes, leading to lysosomal degradation of p62-cargo complexes [21-23].

Paradoxical to the well-known function of p62 as an autophagic adaptor for cell survival, endoplasmic reticulum (ER) stressinduced apoptotic cell death was shown to occur through the ubiquitin-binding function of p62 leading to aggregation of ubiquitinated caspase-8 for subsequent proteolytic autoactivation of caspase-8 [24, 25]. The present study investigated the involvement of p62 in cancer progression and the feasibility of small molecule-mediated targeting of p62 for the therapeutic improvement in therapy-resistant cancer cells using head and neck squamous cell carcinoma (HNSCC) as a model.

HNSCC can be divided into two groups based on the status of human papillomavirus (HPV) infection [26-28]. Although the incidence of $\mathrm{HPV}(+)$ head and neck cancer is rising, tobaccoinduced carcinogenesis remains the leading cause of HNSCC [29-31]. HPV(-) HNSCC patients are less responsive to radiotherapy and have worse survival compared to $\operatorname{HPV}(+) \mathrm{HNSCC}$ patients [32-39]. At present, the mechanism of therapy-resistance in HPV(-) HNSCC is poorly understood. In the current study we found that an increased autophagic flux and apoptosis defects are associated with radioresistance in HPV(-) HNSCC, whereas HPV( + ) HNSCC fail to induce autophagic flux and readily undergo apoptotic cell death upon radiation treatments. We also show that the expression levels of cytosolic p62 proteins are associated with high grade tumors. These results suggest an association of cytoplasmic p62-mediated autophagy with disease progression and therapy resistance. Importantly, pharmacological activation of the p62 ZZ domain using synthetic small molecule ligands promote radiation-induced cytotoxicity via apoptosis induction in therapy-resistant HPV(-) HNSCC. We provide evidence that central to p62-dependent apoptosis in HPV(-) HNSCC is its selfoligomerization along with caspase-8 to form aggresomes-like complexes. Taken together, our study may pave the way for the development of a multimodal treatment to activate apoptosis in a p62-specific manner in intrinsically apoptosis-resistant cells.

\section{MATERIALS AND METHODS \\ Cell culture}

Human HNSCC cell lines UP-SCC-090 and UP-SCC-154 (established at University of Pittsburg), UM-SCC-19 (University of Michigan) and WSU-HN12 (Wayne State University) were cultured as previously described [40].

\section{Antibodies and reagents}

The antibodies used in this study are as follows: mouse monoclonal antip62 (Abcam, ab56416, 1:100,000), mouse monoclonal anti-FK2 specific to Ub-conjugated proteins (Millipore, 04-263, 1:1,000), rabbit polyclonal antiLC3 (Sigma, L7543, 1: 10,000 for immunoblotting; 1:1,000 for immunofluorescence staining), anti-casapse-8 (Santa Cruz, sc-56070 1:2000 for immunoblotting, 1:200 for immunofluorescence staining), rabbit polyclonal anti-GAPDH (Santa Cruz, sc-25778, 1:2,000), rabbit polyclonal anti-GAPDH (BioWorld, AP0063, 1:20,000). The following secondary antibodies were used: alexa fluor 488 goat anti-rabbit lgG (Invitrogen, A11029, 1:200), texas red goat anti-mouse IgG (Invitrogen, T6390, 1:500), anti-rabbit IgG-HRP (Cell Signaling, 7074, 1:10,000), and anti-mouse IgG-HRP (Cell Signaling Technology, 7076, 1:10,000). Other reagents used in this study were bafilomycin A1 (Sigma); high capacity streptavidin agarose resin (Thermo Fisher Scientific).

\section{lonizing radiation treatment (XRT)}

As we previously described [41], cells were irradiated with 0, 2, 4, and 6 Gy using a gantry-mounted Best Theratronics Gammabeam 500 with a dose rate of $1 \mathrm{~Gy} / \mathrm{min}$. Irradiation was carried out at room temperature under atmospheric oxygen conditions. Delivered dose was confirmed with the use of a Farmer chamber.

\section{DEVDase activity assay}

As we previously described [41], cells were lysed with a $0.5 \%$ NP40 lysis buffer, and $50 \mu \mathrm{g}$ of protein lysates was incubated with $10 \mathrm{mmol} / \mathrm{L} \mathrm{Ac}$ DEVD-AMC substrate (Sigma) at $37^{\circ} \mathrm{C}$ for $2 \mathrm{~h}$. Fluorescence was detected using a SpectraMax Gemini (Molecular Probes, Carlsbad, CA) with $360 \mathrm{~nm}$ excitation and $460 \mathrm{~nm}$ emission.

\section{Establishment of p62-knockdown WSU12 cell line}

Scrambled shRNA sequence (shScram; catalog no. RHS4346) and three shRNA against p62 GIPZ (shp62; clone ID no. V3LHS-375194, -375195, -375197) were obtained from Open Biosystems (Huntsville, AL). WSU12 cells were transfected with shScram or p62-targeting shRNA vectors using Lipofectamine 2000 (Invitrogen) and selected with $0.25 \mu \mathrm{g} / \mathrm{mL}$ puromycin. The resulting pooled population were referred to as shp62-94, shp62-95, and shp62-97, respectively.

\section{Immunofluorescence staining}

Human HNSCC cells were cultured on cover slips and fixed with $4 \%$ paraformaldehyde in PBS ( $\mathrm{pH}$ 7.4) for $15 \mathrm{~min}$ at room temperature. After washing three times with PBS, the cells were permeabilized with $0.5 \%$ Triton X-100/PBS solution for $15 \mathrm{~min}$ and washed three times with PBS for $5 \mathrm{~min}$. After three washes with PBS, the cells were incubated with blocking solution (2\% BSA in PBS) for $1 \mathrm{~h}$ and then with primary antibody overnight at $4^{\circ} \mathrm{C}$. Next day, the cells were washed five times for $10 \mathrm{~min}$ each time with PBS and then incubated with secondary antibody for $1 \mathrm{~h}$. The cells were washed five times with PBS for 10 min each time, and DAPI stained for $10 \mathrm{~min}$. After three washes with PBS, the coverslips were mounted on slides using the coverslips Fluoro-GEL (Electron Microscopy Sciences). Confocal images were taken by laser scanning confocal microscope 510 Meta (Zeiss) and analyzed using Zeiss LSM Image Browser (ver. 4.2.0.121).

\section{Immunoprecipitation assay}

WSU12 cells, treated with vehicle control (DMSO) or YOK1104 for $12 \mathrm{~h}$ in the presence or absence of XRT ( $6 \mathrm{~Gy}$ ), were lysed in binding buffer [ $20 \mathrm{mM}$ Tris- $\mathrm{HCl}, \mathrm{pH} 7.6,125 \mathrm{mM} \mathrm{NaCl}$, and $1 \%$ Nonidet P-40 with a cocktail of protease and phosphatase inhibitors (Roche)] through one cycle of freezing in liquid nitrogen and thawing in a $42{ }^{\circ} \mathrm{C}$ water bath. Genomic DNA was sheared by passing the extracts through a 26-gauge needle four times. The resulting lysates were centrifuged at $3000 \times g$ for $15 \mathrm{~min}$ to pellet the cellular debris. Total $500 \mu \mathrm{g}$ proteins were incubated with $2 \mu \mathrm{g}$ control IgG or mouse monoclonal antibody raised against full-length recombinant caspase-8 of human origin (Santa Cruz, sc-56070) for overnight, followed by incubation with $40 \mu$ l of $50 \%$ protein A agarose bead slurry for $1 \mathrm{~h}$. The beads were washed with the binding buffer for 5 min at $4{ }^{\circ} \mathrm{C}$ with gentle agitation; washing was repeated three times. The proteins bound to protein $A / G$ agarose beads were dissociated in 2X SDS sample buffer, heated at $100{ }^{\circ} \mathrm{C}$ for $5 \mathrm{~min}$, and separated on a SDS-PAGE. For immunoblot analysis of caspase- 8 using caspase- 8 IP products, the secondary HRP antibody (GeneTex, GTX221667-01), which specifically reacts with the native, non-reduced form of mouse IgG, was used to distinguish the caspase-8 band from immunoglobulin heavy chain bands.

\section{p62 immunohistochemistry}

HNSCC blocks were retrieved from the Pathology core at Wayne State University and sections were deparaffinized and then hydrated. Microwave heat-induced antigen retrieval was performed using the Antigen Citrus Plus Retrieval Solution (BioGenex, HK081-5K). Non-specific binding sites were blocked with $2.5 \%$ normal horse serum in a wet chamber at $4{ }^{\circ} \mathrm{C}$, overnight. p62 (AbCam, ab56416) was diluted 1:2000 in blocking solution then incubated in a wet chamber at $4{ }^{\circ} \mathrm{C}$ for $6 \mathrm{~h}$. The ImmPRESS ${ }^{\text {TM }}$ HRP AntiMouse IgG (Peroxidase) Polymer Detection Kit (Vector Laboratories, MP7402) and the ImmPACT ${ }^{\mathrm{TM}}$ DAB Peroxidase Substrate (Vector Laboratories, SK-4105) were used to develop the signal of stained slides followed by counterstaining with Mayer's Hematoxylin (Scytek, HMM999). Images were obtained using a Zeiss Axioplan 2 microscope. Scoring was performed by a 
pathologist using a scale of $0=$ no staining, $1=$ low, $2=$ moderate, $3=$ high stain.

\section{Proximity Ligation Assay (PLA)}

The proximity ligation assay was performed according to the manufacturer's protocol using the Duolink ${ }^{T M}$ In Situ Red Starter Kit Mouse/Rabbit (Sigma-Aldrich). Deparaffinization, hydration, and antigen retrieval were performed as described in the IHC section. p62 (AbCam, ab56416) and LC3 (Cell Signaling Technology, 2775) were diluted 1:2000 in antibody diluent overnight in a humidity chamber at $4{ }^{\circ} \mathrm{C}$. Images were obtained using a Leica DMi3000 B Fluorescence Microscope.

\section{Statistical analysis}

All experiments were repeated at least three times, and all data are presented as the mean \pm SD. Statistical analysis was performed using Student's unpaired two-tailed $t$-test or Chi-square test whose significance was determined according to $p$ values $\left(p<0.001\left(^{* * *}\right), p<0.01\left(^{* *}\right)\right.$, $p<0.05(*))$.

\section{RESULTS}

Activation of the autophagy-lysosome pathway is a marker of radioresistance in HPV(-) HNSCC

To understand the molecular mechanisms of radioresistance in HNSCC cells, we assessed the relationship between autophagic activities and radiosensitivity in HPV(-) HNSCC cells (WSU12 and UM19) compared to HPV( + ) HNSCC cells (UP090 and UP154). The HPV status in these cell lines was confirmed by RT-PCR analyses of the HPV oncogenes E6 and E7 (Supplementary Fig. 1a). Consistent with previous reports [32-39], HPV(-) WSU12 and UM19 cells were more resistant to radiation treatment than $\operatorname{HPV}(+)$ UP090 and UP154 cells (Fig. 1a). Upon radiation treatment, apoptotic cell death was evident in HPV $(+)$ HNSCC cells as determined by caspase activation (Fig. 1b) whereas no apoptosis was observed in HPV(-) HNSCC cells.
Next, we examined autophagic activity by monitoring the synthesis of LC3 and its lipidation to LC3-II. Immunoblotting showed that the expression level and lipidation of LC3 were higher in HPV(-) HNSCC cells in comparison with HPV( + ) HNSCC cells (Fig. 1c), suggesting that HPV inhibits autophagy in host cells through an unknown mechanism. A strong accumulation of LC3-II was observed in HPV(-) HNSCC cells treated with bafilomycin A1, an inhibitor of the lysosomal proton pump V-ATPase that inhibits autophagosome-lysosome fusion (Fig. 1d, e). These results indicate that the increases of LC3-II in HPV(-) HNSCC cells are indeed due to enhanced autophagic flux which was not observed in $\mathrm{HPV}(+)$ HNSCC cells (Fig. 1d, e). To confirm differential autophagic flux between HPV $(-)$ and HPV $(+)$ HNSCC in vivo, we visualized the targeting of $\mathrm{p} 2^{+}$to $\mathrm{LC}^{+}$autophagic membranes by the proximity ligation assay (PLA) that detects the colocalization of two proteins within the distance of $40 \mathrm{~nm}$. Using antibodies against $\mathrm{p} 62$ and LC3 the PLA readily revealed a number of $\mathrm{p} 62^{+} \mathrm{LC}^{+}$autophagic membranes in HPV(-) HNSCC tissues, whereas this complex was barely detected in $\operatorname{HPV}(+)$ HNSCC tissues (Fig. 1f, g). These results demonstrate that therapyresistant HPV(-) HNSCC cells have higher levels of autophagic flux relative to $\mathrm{HPV}(+)$ HNSCC cells.

\section{The cytosolic levels of p62 correlates with the progression of HNSCC}

In autophagy, cargoes are selectively recognized and collected by autophagic cargo receptors such as p62 and other Sequestosomelike receptors (SLRs) [42]. Recent studies have suggested a functional and/or progonstic significance of cytosolic vs. nuclear p62 in tumor progression and therapeutic responses [43, 44]. We therefore examined the subcellular localization of p62 in HNSCC tissues. The specificity of p62 antibody was confirmed using control and p62 knockdown HPV(-) WSU cells (Supplementary Fig. 1b). The staining intensity of nuclear and cytoplasmic p62 in HNSCC tissues was evaluated by a pathologist and scored on a a

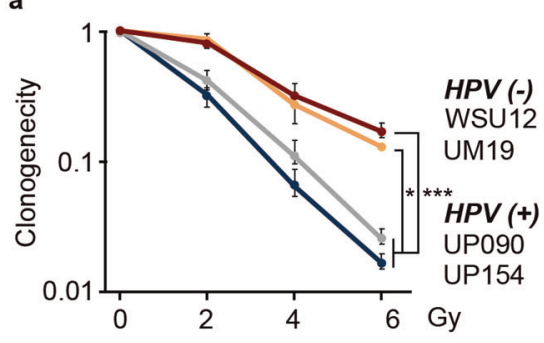

b

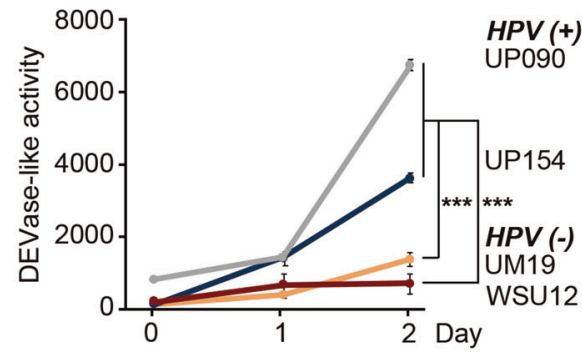

f

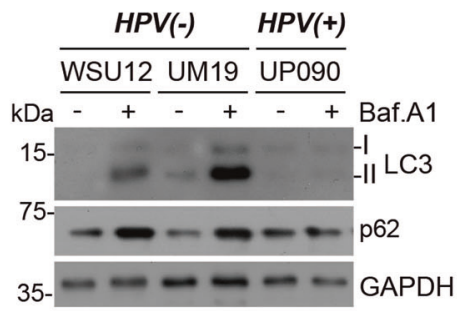

e

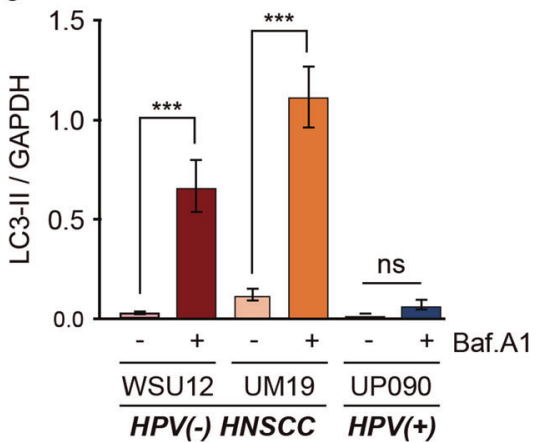

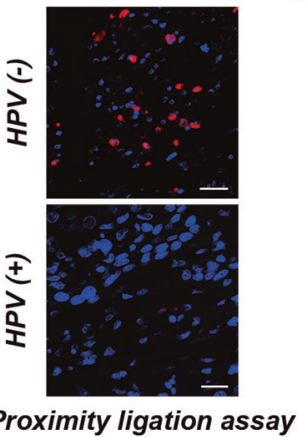

C

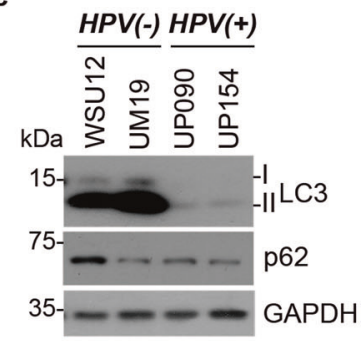

g

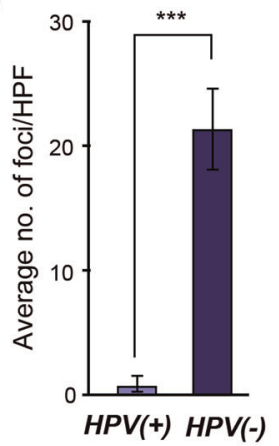

Fig. 1 Defective apoptosis and increased autophagic flux in radioresistant HPV(-) HNSCC. a Clonogenic cell survival assay of HPV $(+)$ HNSCC cell lines (UP090 and UP154) and HPV(-) HNSCC (WSU12 and UM19) upon ionizing radiation (XRT) $\left({ }^{*} p<0.05,{ }^{* * *} p<0.001\right)$. b DEVDase (caspase-3/caspase-7) activity assay in HNSCC cells at indicated time points post irradiation at 6 Gy $\left(^{* * *} p<0.001\right)$. c Immunoblotting analysis of LC3 or GAPDH in HPV(-) HNSCC cell lines (WSU12 and UM19) and HPV( + ) HNSCC cell lines (UP090 and UP154). d Autophagic flux assay using WSU12, UM19 or UP154 cells in the absence or presence of bafilomycin A ( $500 \mathrm{nM}, 6 \mathrm{~h})$. e Quantification of LC3-II band intensity of d. Error bars represent means \pm SD from three independent experiments $(* * *<<0.001$, ns: non-significant). $f$ In situ detection of autophagosomes in HPV(-) and HPV ( + ) HNSCC tissues by p62 and LC3 PLA. Scale bar: $30 \mu \mathrm{m}$. g The average number of positive foci in p62 and LC3 PLA quantitated at $10 \mathrm{X}$ magnification $(n=4, * * * 0<0.001)$. 
a
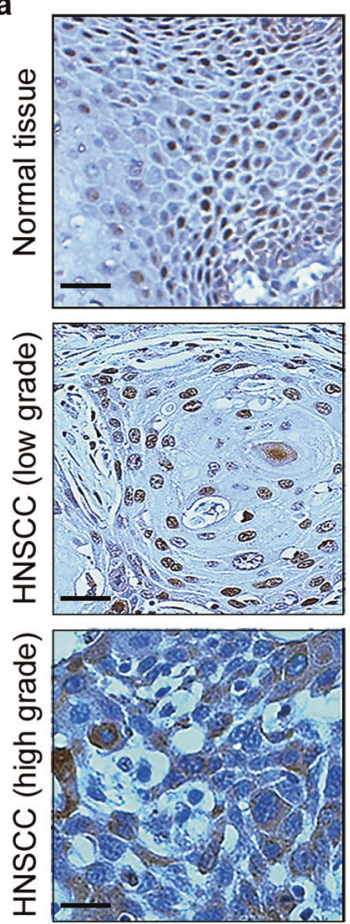

b

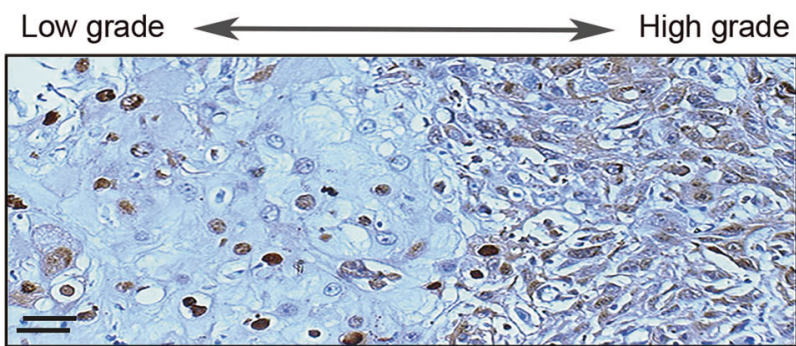

C

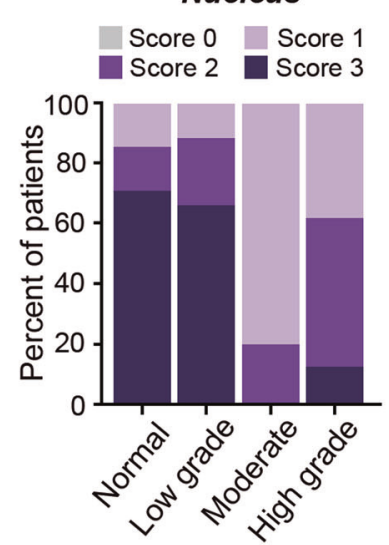

d

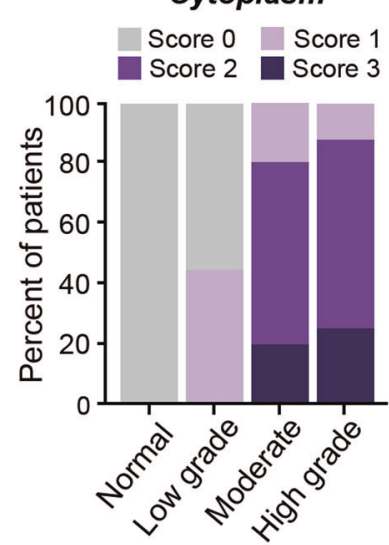

e

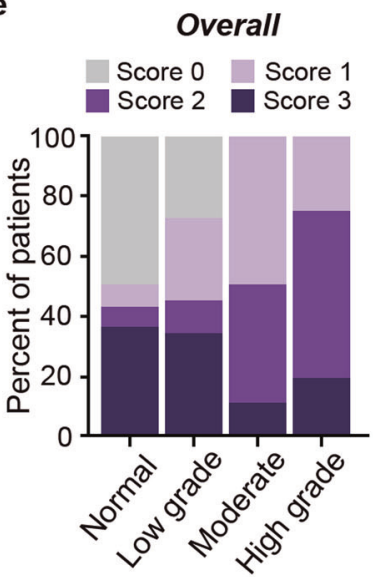

Fig. 2 Cytosolic p62 is associated with advanced HNSCC. a Immunohistochemical analysis of p62 in normal tissue (top), low grade (middle), and high grade (bottom) HNSCC. Scale bar, $50 \mu \mathrm{m}$. b Immunohistochemical analysis of cytosolic and nuclear p62 localization corresponding to tumor progression. Scale bar, $50 \mu \mathrm{m}$. c Frequency of nuclear p62 in normal tissues and low, moderate, and high-grade HNSCC as assessed by expression levels (score 0-3), analyzed by Chi-square test, $p=0.025$. d Frequency of cytoplasmic p62 in normal tissues and low, moderate, and high-grade HNSCC as assessed by expression levels (score $0-3$ ), analyzed by Chi-square test, $p<0.001$. e Frequency of overall p62 in normal tissues and low, moderate, and high-grade HNSCC as assessed by expression levels in both nucleus and cytoplasm (score 0-3), analyzed by Chi-square test, $p=0.001$.

scale of 0 to 3. Typical immunostaining patterns of $\mathrm{p} 62$ in normal tissues, HPV(-) low grade, and HPV(-) high-grade HNSCC are shown in Fig. 2a. Interestingly, cytoplasmic translocation of p62 was associated with disease progression within the same patient tissue (Fig. 2b). An analysis of 7 normal tissue, 9 low grade, 5 moderate, and 8 high-grade HNSCC specimens showed that p62 was almost exclusively found in the nucleus of normal tissues and low-grade HNSCC (Fig. 2c). However, the localization of p62 gradually shifted from the nucleus to the cytosol as the tumors advanced into moderate and high-grade HNSCC (Fig. 2c, d). The overall expression levels of p62 was also associated with higher tumor grade (Fig. 2e). Collectively, these results showed association between the cytosolic localization of p62 and HPV(-) HNSCC tumor progression, consistent with a previous report that increased LC3 puncta, high cytoplasmic p62, and low nuclear p62 expressions are associated with poor prognosis and aggressive clinicopathologic features of oral squamous cell carcinoma (OSCC) [43].

\section{Synthetic small-molecule ligands of p62 induce autophagic flux in HPV(-) HNSCC cells}

We previously developed a small-molecule ligand, YOK1104, to the ZZ domain of p62 that induces self-polymerization of p62 and p62 interaction with LC3 on autophagic membranes [22]. Given the correlation between cytoplasmic p62 and HNSCC progression and the association between autophagic flux and radioresistance, we tested whether YOK1104 can modulate radiosensitivity of HPV (-) HNSCC cells. We first confirmed that YOK1104 binds to the ZZ domain of p62 using streptavidin pulldown assay with biotinylated YOK1104 (Fig. 3a-d). Next, we tested whether YOK1104 can affect p62-mediated autophagic flux in HPV(-) and HPV( + ) HNSCC cells. Immunofluorescence analyses of HPV(-) HNSCC cells treated with YOK1104 revealed a drastic increase in the formation of cytosolic p62 and LC3 puncta, which showed strong colocalization to form $\mathrm{p} 62^{+} \mathrm{LC}^{+}$double positive cytosolic puncta (Fig. 3e). The formation of $\mathrm{p} 2^{+} \mathrm{LC}^{+}$puncta was associated with the increases in the synthesis and lipidation of LC3 (Fig. 3f) as well as the colocalization of LC3 puncta with the cytosolic puncta positive for LAMP1, a lysosomal marker (Fig. 3g). Consistent with increased autophagic flux, YOK1104 enhanced the formation of cytosolic puncta positive for Beclin1, indicative of enhanced autophagosomal nucleation (Fig. 3h). These results show that the smallmolecule ligand YOK1104 increases the activity of p62 in macroautophagy, autophagosome biogenesis, and autophagic flux in HPV(-) HNSCC cells. In contrast, YOK1104 exerted no such autophagy-inducing activity in $\mathrm{HPV}(+) \mathrm{HNSCC}$ cells as evidenced by the minimal levels of LC3-II (Fig. 3f). Likewise, neither the formation of cytosolic puncta positive for LC3 (Fig. 3i, j), the colocalization of $\mathrm{LC}^{+}$autophagosomes with LAMP1 ${ }^{+}$lysosomes (Fig. 3k, I) nor Beclin1 (Fig. 3m) was found in HPV( + ) HNSCC cells treated with YOK1104. These results show that YOK1104 induces p62-dependent autophagy in HPV(-) HNSCC but not in autophagydefective HPV $(+)$ HNSCC cells.

\section{YOK1104 enhances radiosensitivity of therapy-resistant HPV (-) HNSCC cells via apoptosis induction in a p62-dependent manner}

Accumulating evidence suggests that autophagy is associated with therapy-resistance $[8,9]$. Besides the role of p62 in autophagy, recent studies demonstrated multi-functions of p62 including its role in caspase-8 activation [24, 25]. Here, we examined whether YOK1104 activation of p62 further increases therapy-resistance via its induction of autophagic flux or by 
a

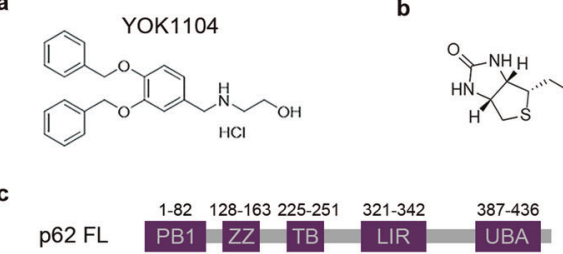

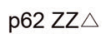

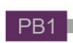

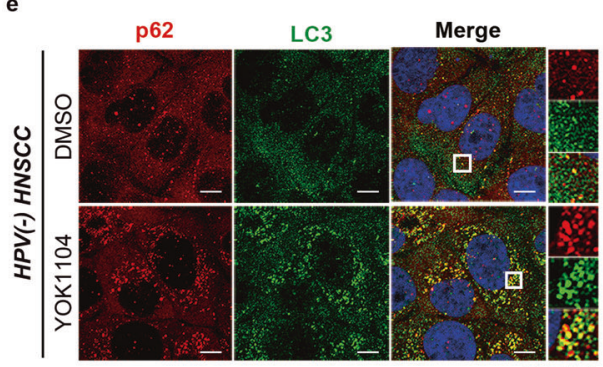

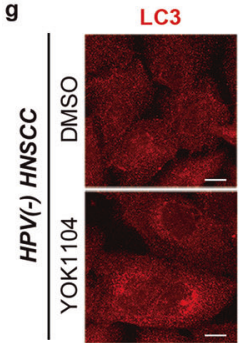

LAMP1

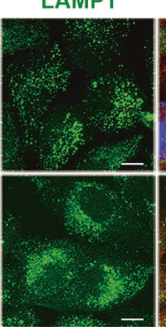

h

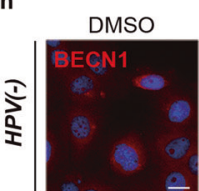

Merge

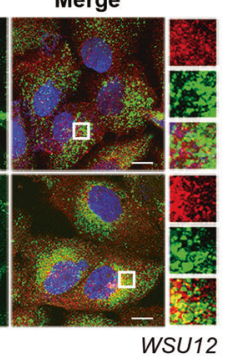

WSU12

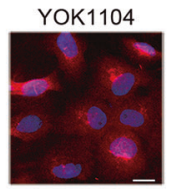

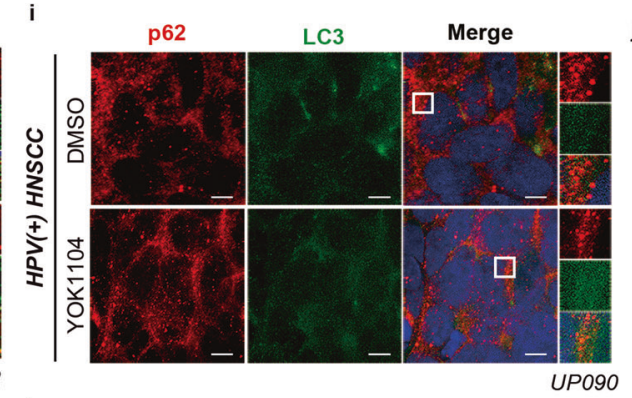

k

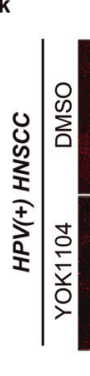

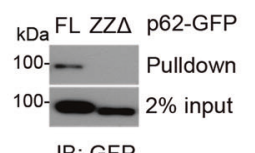

IB: GFP in vitro YOK1104-Biotin pulldown assay f

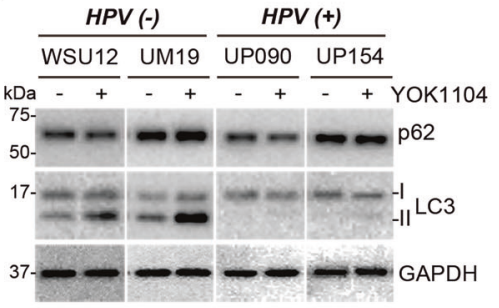

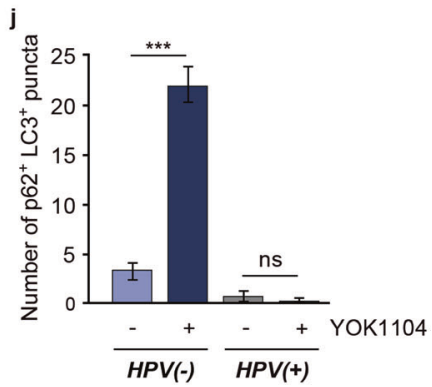
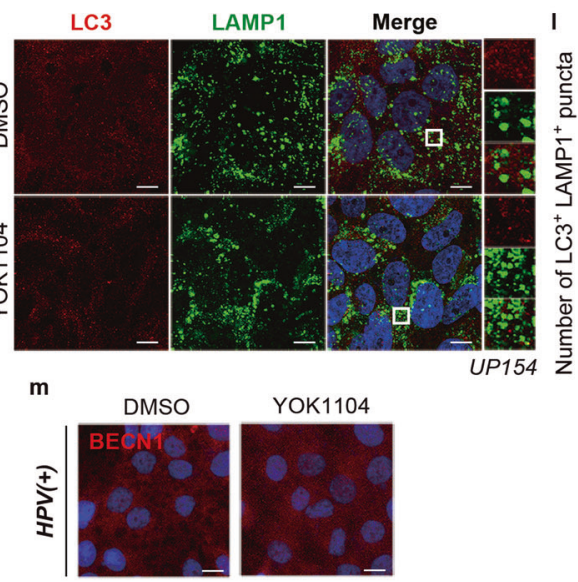

YOK1104
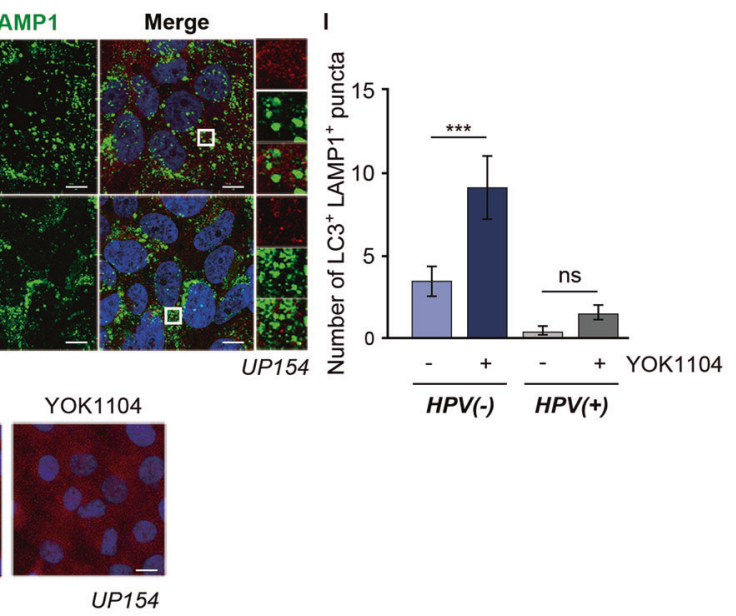

Fig. 3 p62 ligand YOK1104 induces autophagy in radioresistant HPV(-) HNSCC. a, b Chemical structures of free or biotinylated p62-ZZ small-molecule ligand YOK1104. c Schematic illustration of full-length and ZZ domain-deleted mutant p62 constructs. d In vitro YOK1104Biotin pulldown assay using full-length p62-GFP or $\Delta$ ZZ-p62-GFP proteins, purified from HEK293 cells after transient transfection of those expression vectors. e Immunostaining of p62 (red) and LC3 (green) in WSU12 HPV(-) HNSCC treated with vehicle control (DMSO) or $5 \mu$ M YOK1104. Scale bar: $10 \mu \mathrm{m}$. f Immunoblot analysis of p62, LC3, or GAPDH with or without YOK1104 treatment. g Immunostaining of LC3 (red) and LAMP1 (green) in WSU12 HPV(-) HNSCC treated with vehicle control (DMSO) or $5 \mu$ M YOK1104. Scale bar: $10 \mu \mathrm{m}$. $\mathbf{h}$ Immunostaining of Beclin1 (red) in UM19 HPV(-) HNSCC treated with vehicle control (DMSO) or $5 \mu$ M YOK1104. Scale bar: $20 \mu \mathrm{m}$. i Immunostaining of p62 (red) and LC3 (green) in UP090 HPV( + ) HNSCC treated with vehicle control (DMSO) or $5 \mu$ M YOK1104. Scale bar: $10 \mu \mathrm{m}$. $\mathbf{j}$ Quantification of colocalization (yellow) of p62 with LC3 in e and $\mathbf{i}\left(n=20\right.$ cells) ${ }^{* * *} p<0.001$, ns non-significant). $\mathbf{k}$ Immunostaining of LC3 (red) and LAMP1 (green) in UP154 HPV (+) HNSCC treated with vehicle control (DMSO) or $5 \mu \mathrm{M}$ YOK1104. Scale bar: $10 \mu \mathrm{m}$. I Quantification of colocalization (yellow) of LC3 with LAMP1 in $\mathbf{g}$ and $\mathbf{k}$ ( $n=20$ cells) ${ }^{* * *} p<0.001$, ns: non-significant). $\mathbf{m}$ Immunostaining of Beclin1 (red) in UP154 HPV( + ) HNSCC treated with vehicle control (DMSO) or $5 \mu$ M YOK1104. Scale bar: $20 \mu \mathrm{m}$.

converting its signal to programmed cell death. To this end, we first determined the effects of YOK1104 treatments on radiotherapy-induced cytotoxicity in HNSCC cells. YOK1104 rendered intrinsically radio-resistant HPV(-) UM19 and WSU12 cells sensitive to ionizing radiation (Fig. 4a, b). Notably, the radiosensitizing efficacy of YOK1104 on HPV(-) WSU12 and UM19 cells was achieved at a dose as low as $2 \mathrm{~Gy}$, a clinically relevant dose. Unlike HPV(-) HNSCC cells, YOK1104 had little effect on survival of autophagy-defective HPV(+) UP090 and UP154 cells (Fig. 4a). Moreover, the efficacy of YOK1104 was higher in UM19 cells with higher autophagic flux compared to WSU12 cells (Fig.1d and Supplementary Fig. 1c-e). These results suggest a positive correlation between the ability of YOK1104 to promote radiotoxicity and the levels of cells' intrinsic autophagic flux. To confirm that YOK1104-mediated cytotoxicity is dependent of p62, WSU12 cells were engineered to stably express shRNA against p62 (Fig. 4c). As shown in Fig. 4b, p62 knockdown virtually abolished the ability of YOK1104 to enhance radiotoxicity, demonstrating the specificity of YOK1104 to p62.

Next, we determined whether YOK1104-enhanced radiotoxicity in HPV(-) HNSCC cells involves apoptotic cell death. Neither YOK1104 treatment nor ionizing radiation up to $6 \mathrm{~Gy}$ alone was sufficient to activate DEVDase, reflecting activation of procaspases 3 and 7 (Fig. 4d). However, combined treatments with radiation and YOK1104 induced caspase activity, which was effectively counteracted by p62 knockdown (Fig. 4d). These results 
a

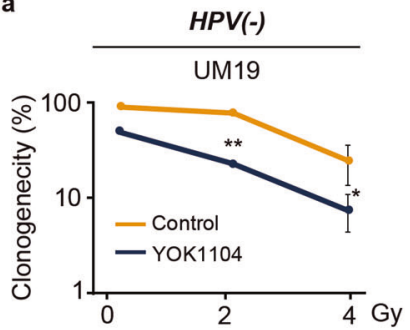

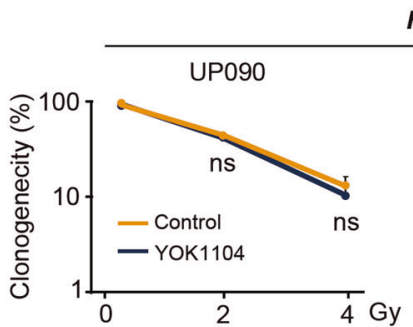

HPV(+)

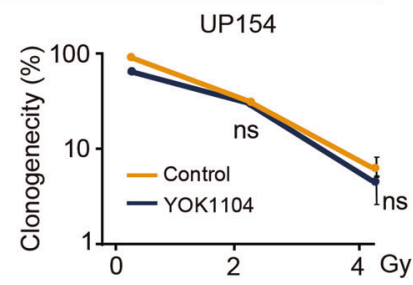

b WSU12 HPV(-) HNSCC
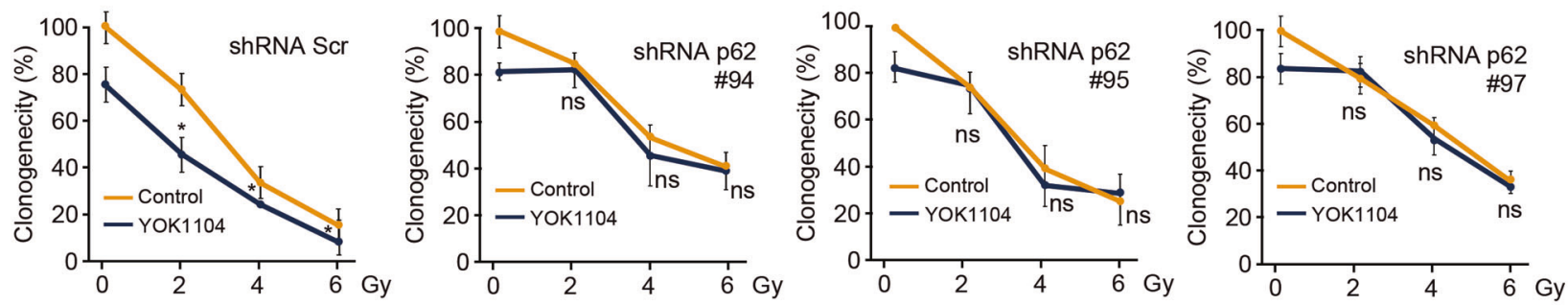

c

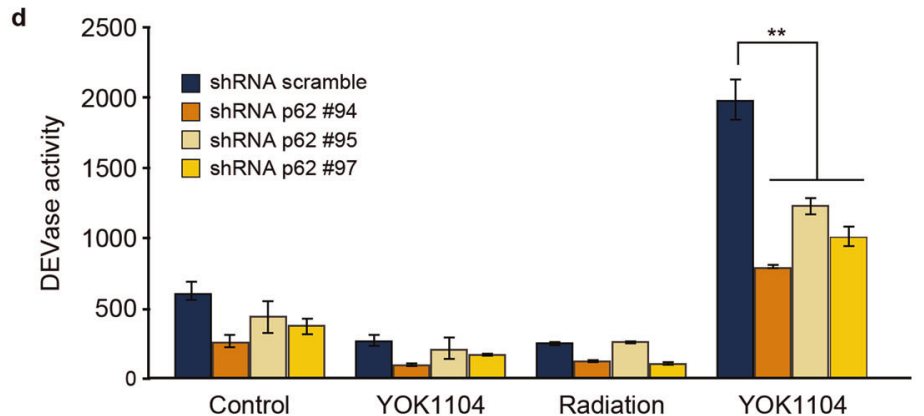

Fig. 4 YOK1104-mediated p62 activation promotes radiotoxicity in HPV(-) via caspase activation in a p62-dependent manner. a

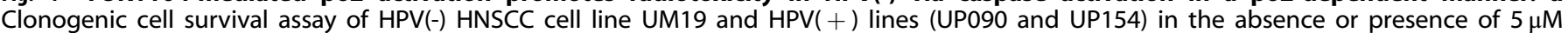

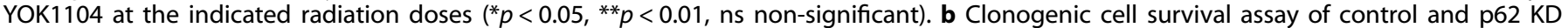

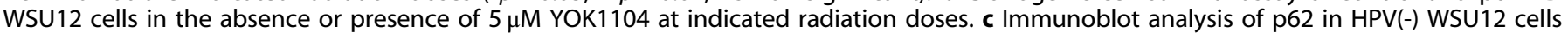

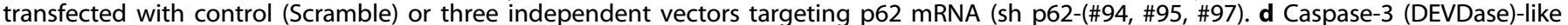

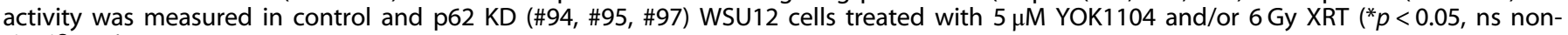
significant).

suggest that YOK1104-activated p62 induces apoptotic cell death in intrinsically therapy-resistant and apoptosis-defective HNSCC cells upon ionizing radiation treatment.

\section{YOK1104-activated p62 induces the formation of aggresome- like caspase-8 complex}

We investigated whether pharmacological activation of p62 induces apoptotic cell death through the modulation of autophagic flux and/or caspase-8 in HPV(-) HNSCC cells upon irradiation. As expected, YOK1104 treatment enhanced autophagic flux in HPV(-) HNSCC cells as indicated by increased LC3 lipidation in the presence of bafilomycin A1 (Fig. 5a-d, lanes 4 vs. 3). It should be noted that immunoblots with shorter exposure time are shown to examine the accumulation of LC3-II and p62 in the presence of bafilomycin following treatments with YOK1104 and/or radiation. Strikingly, such autophagic flux was drastically reduced by irradiation (Fig. 5a-d, lanes 6 vs. 5). YOK1104-induced LC3-II and p62 accumulations, detected by bafilomycin treatment, was decreased upon irradiation (Fig. 5a-d, lanes 8 vs. 4). This indicates that the autophagy system is downregulated by co-treatment with YOK1104 and radiation. Thus, the p62 ZZ ligand YOK1104promoted radiotoxicity is unlikely due to its sudden induction of autophagic flux at a toxic level.

Caspase-8 is an initiator caspase, pivotal in the extrinsic pathway of apoptosis $[45,46]$. Death ligand binding to its cognate receptors on the plasma membrane induces the formation of the death-inducing signaling complex (DISC) where caspase- 8 is recruited and activated $[45,46]$. The E3 ligase Cullin3, that is associated with DISC, polyubiquitinates caspase-8 which subsequently interacts with the UBA domain of p62. These interactions promote aggregation and full activation of caspase-8. Subsequently, p62 transports the activated caspase-8 to cytosolic ubiquitin-rich aggresomes, which in turn induce apoptotic signaling pathways [25, 47]. Here, we examined whether YOK1104-activated p62 in combination with ionizing radiation activates the ubiquitinated caspase- 8 , similarly to caspase- 8 activation in the extrinsic pathway. Immunoblot analyses detected an active (cleaved) form of caspase-8 in apoptosis-prone $\mathrm{HPV}(+)$ UP154 cells upon irradiation (Supplementary Fig. 2a), but not in apoptosis-resistant HPV(-) WSU12 cells (Fig. 5e). While YOK1104 treatment alone had no effect on caspase-8 activation, cotreatment with radiation resulted in caspase-8 activation in HPV (-) HNSCC cells (Fig. 5e). Moreover, caspase3/7 activity induced by co-treatment was abolished in the presence of caspase- 8 inhibitor (Fig. 5f). These results indicate the functional significance of caspase-8 as an initiator of caspase cascade upon YOK1104 and radiation treatments.

Next, we monitored the ubiquitination of caspase-8 in HPV(-) WSU12 cells treated with irradiation and/or YOK1104. The ubiquitination of caspase- 8 was prominent upon irradiation alone but not by YOK1104 (Fig. 5g, right panel). A control experiment for the specificity of caspase- 8 immunoprecipitation is shown in 

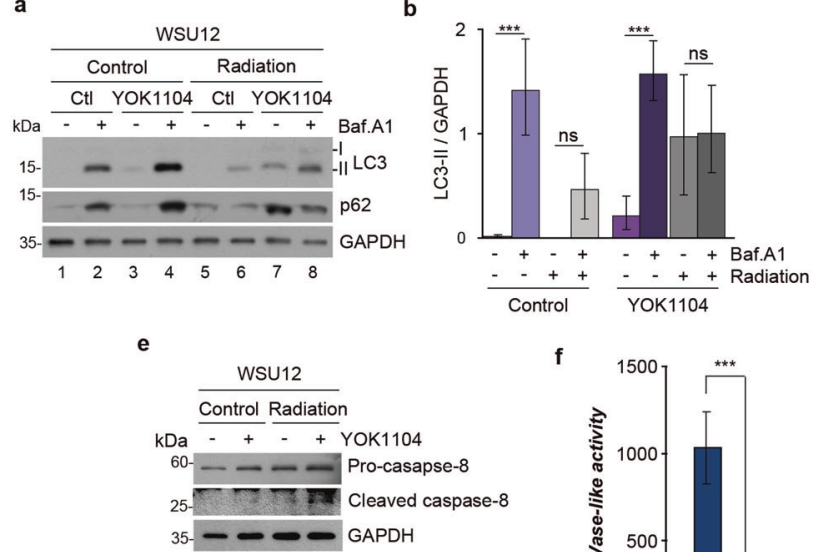

h
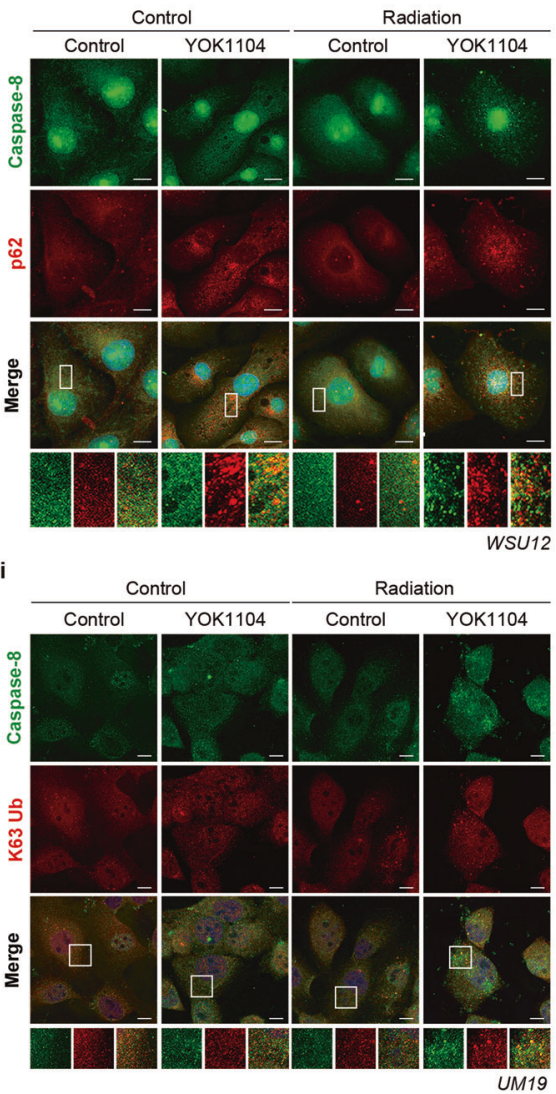
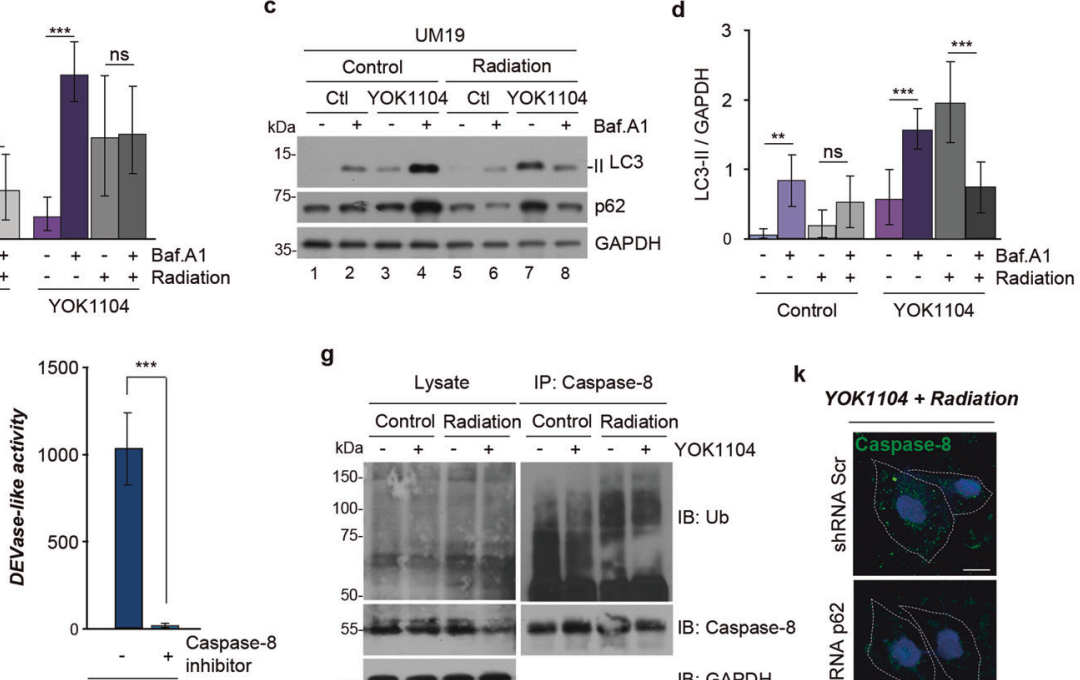

YOK1104/ Radiation

j

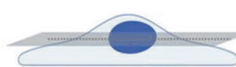

k

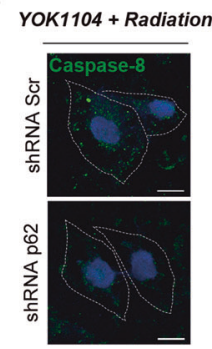

WSU12

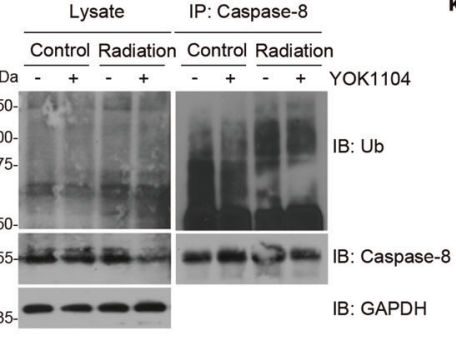

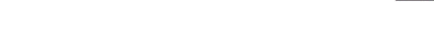

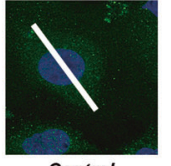

Control

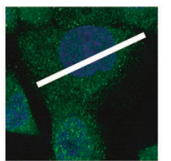

YOK1104

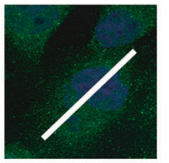

Radiation

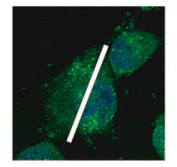

Radiation/YOK1104

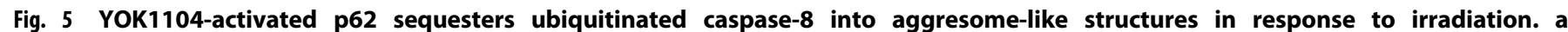

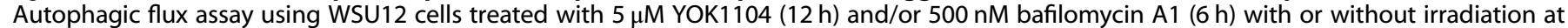

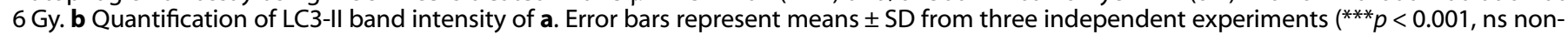

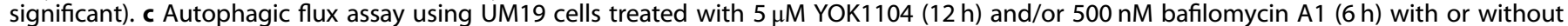

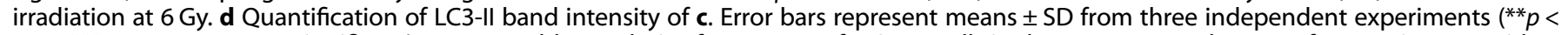

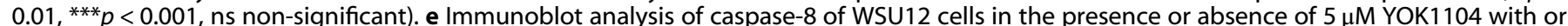

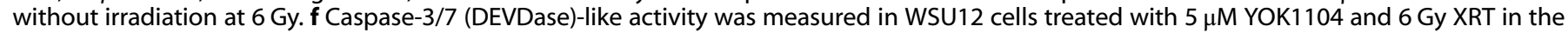

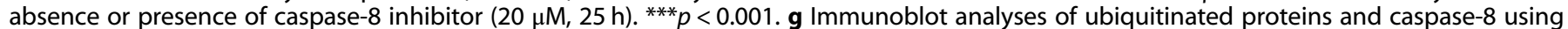

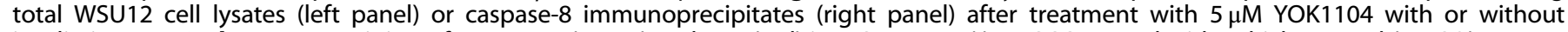

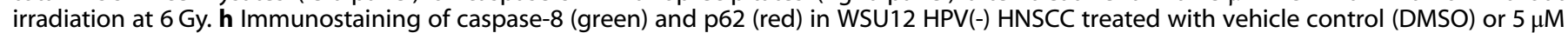

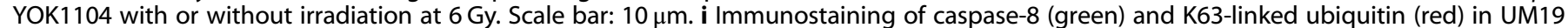

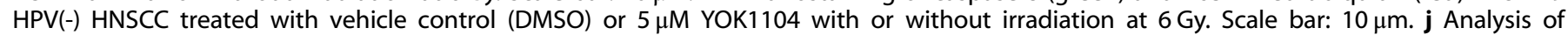

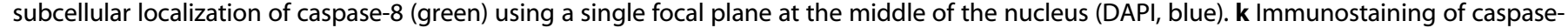
8 (green) in control and p62 KD WSU12 HPV(-) HNSCC treated with $5 \mu \mathrm{M}$ YOK1104 and irradiation at 6 Gy. Scale bar: $10 \mu$ m.

Supplementary Fig. 2b. We then asked whether YOK1104-bound p62 facilitates the formation of caspase-8 aggresomes in HPV(-) HNSCC cells after irradiation. Immunostaining analyses of caspase8 and p62 did not detect caspase- 8 aggresomes when treated with either irradiation or YOK1104 alone (Fig. 5h). Strikingly, a large number of caspase- 8 aggresomes were generated by cotreatment with radiation and YOK1104 (Fig. 5h). Moreover, we detected colocalization of caspase- 8 with K63-linked ubiquitin 


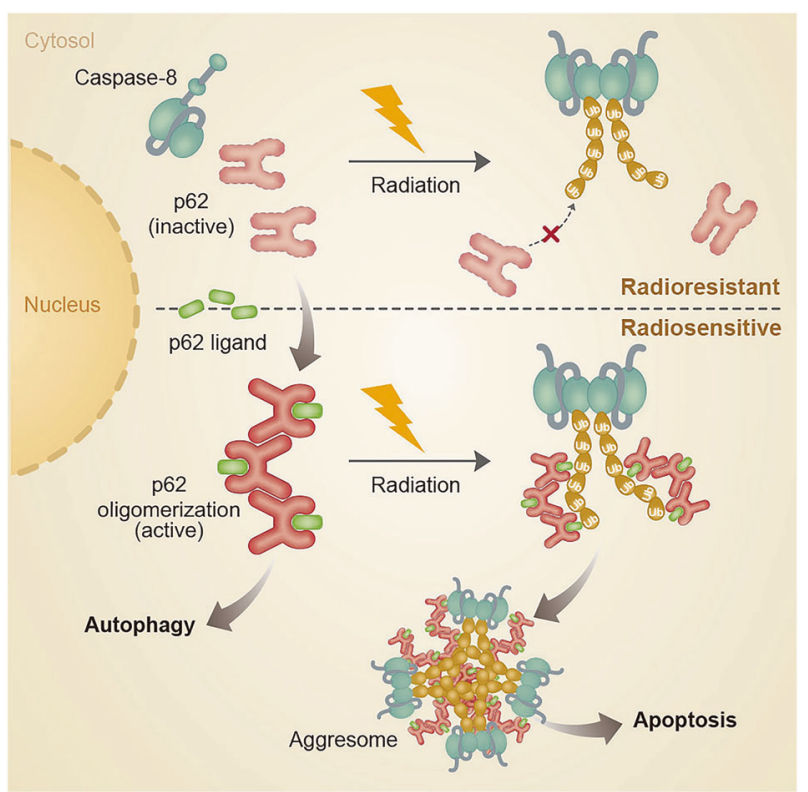

Fig. 6 A graphical illustration of p62-dependent caspase-8 activation upon irradiation. Synthetic p62-ZZ ligands, such as YOK1104, induce p62 self-oligomerization, its interaction with LC3, and autophagosome biogenesis. lonizing radiation treatment induces ubiquitination of caspase-8. A synthetic ligand-activated p62 via its ZZ domain promotes aggregation of ubiquitinated caspase-8 into aggresomes-like structures leading to apoptosis.

chains, further supporting the formation of caspase- $8^{+}$aggresomes by co-treatment with YOK1104 and radiation (Fig. 5i). Confocal microscopy revealed that caspase-8 puncta, generated after YOK1104/radiation co-treatment, were present mostly in the cytoplasm including the perinuclear region (Fig. 5j). Importantly, the formation of caspase-8 puncta was abolished when p62 was downregulated in WSU12 cells (Fig. 5k). Immunofluorescence staining analyses showed that while treatment with YOK1104 alone increased $\mathrm{LC}^{+} \mathrm{p} 62^{+}$puncta, co-treatment with YOK1104 and radiation reduced $\mathrm{LC}^{+} \mathrm{p}^{+} 2^{+}$puncta and increased caspase$8^{+} \mathrm{p}^{+} 2^{+}$puncta (Supplementary Fig. $2 \mathrm{c}-\mathrm{e}$ ). Taken together, these results demonstrate that while YOK1104 promotes autophagic flux, co-treatment with radiation exerts its cytotoxic effect by reducing autophagic flux and, in parallel, inducing apoptotic cell death mediated by caspase- 8 and $p 62$.

In conclusion, this study suggests that irradiation induces ubiquitination of caspase-8 by an as-yet-unknown mechanism, and ubiquitination of caspase- 8 itself is insufficient to interact with p62 for the formation of aggresome. Importantly, pharmacologic activation and oligomerization of $\mathrm{p} 62$ provides a focal point for caspase-8 aggregation leading to caspase-8 activation (Fig. 6). Thus, we propose that small-molecule ligands to the $062 \mathrm{ZZ}$ domain have therapeutic potential as radiosensitizers in intrinsically apoptosis-resistant HPV(-) HNSCC.

\section{DISCUSSION}

In this study, we investigated the mechanisms underlying radioresistance in HPV(-) HNSCC and examined the potential of p62 as a druggable target to improve the efficacy of radiation therapy. To this end, we first characterized the distinct responses of HPV $(+)$ vs. HPV(-) HNSCC to ionizing radiation and observed that the latter were not only less susceptible to radiotoxicity but also resistant to caspase activation. HPV(-) HNSCC exhibited both drastically greater levels of autophagosomes and their accelerated turnover. In association with this, our study suggests that the cytosolic level of p62 and cellular level of autophagy flux correlate with the radioresistance and the progression of HPV(-) HNSCC. We explored whether this pro-survival autophagic pathway can be targeted by novel therapies. A small-molecule ligand to the $Z Z$ domain of p62, termed YOK-1104, was successfully used to induce p62 self-oligomerization, autophagic sequestration of its cargoes, and autophagosome biogenesis in $\mathrm{HPV}(-)$, in sharp contrast to HPV $(+)$ HNSCC. Such structural and functional activation of $p 62$ by a synthetic ligand sensitized HPV(-) HNSCC to radiation-induced radiotoxicity. Mechanistically, irradiation of HPV(-) HNSCC results in ubiquitination of caspase-8, which appears to be a prerequisite but insufficient for its interaction with p62. Importantly, pharmacologic activation of p62 facilitates caspase $-8^{+}$p $62^{+}$aggresomelike structures for the activation of the subsequent apoptotic signaling cascade. Our finding that synthetic ligand-mediated p62 activation promotes radiotoxicity via induction of apoptosis in cells with high autophagic flux is particularly significant since this approach may induce malignant cancer-specific cell death with less normal cell toxicity.

Interestingly, HPV(-) HNSCC cells not only exhibited higher basal degrees of autophagy flux and p62-LC3 interaction as opposed to their $\mathrm{HPV}(+)$ counterparts, but also were more readily responsive to p62-ZZ ligand-mediated autophagy induction. Our results showed that synthetic activation of p62 via its $Z Z$ domain primes $\mathrm{HPV}(-)$, but not $\mathrm{HPV}(+)$, HNSCC for radiation-induced cell death by sequestering ubiquitinated caspase- 8 into aggresome-like structures. Failure of p62 activation in $\operatorname{HPV}(+)$ cells would hinder the self-oligomeric activity of p62 and the ability to sequester its cargoes, including caspase-8. Given the significance of p62regulated ER stress signaling in the regulation of both autophagy and programmed cell death, it would be of importance to investigate functional relationship between radiosensitivity and misregulation of p62 and/or the N-degron pathway in the future. Interestingly, photodynamic therapy (PDT) directed at the ER/ mitochondria using benzoporphyrin derivative (BPD) synergized with YOK1104 for caspase activation whereas PDT using NPe6, a selective photosensitizer for lysosomes, failed to do so (Supplementary Fig 3).

Upon death signals, the E3 ligase Cullin3 polyubiquitinates autoprocessed caspases-8 catalytic domain fragments, which in turn recruits p62 to the ubiquitinated chains. p62 transports the liberated catalytic domain of casepase- 8 to ubiquitin-rich "aggresomes" in the cytosol [25]. A pertinent question in our study is why ubiquitinated caspase-8 fails to form aggresomes with p62 in HPV (-) HNSCC cells upon irradiation. We surmise that in the absence of adaptor proteins such as FADD in DISC, monomers of caspase-8 and p62 fail to interact with each other in the formation of aggresome. Importantly, our study demonstrated that YOK1104induced oligomerization and activation of p62 are sufficient to initiate the formation of caspase $-8^{+} \mathrm{p} 62^{+}$puncta upon cotreatment with radiation even in the absence of death receptor activation. It would be of importance to determine the subcellular location where YOK1104-activated p62 oligomers initiate aggresome formation with caspase- $8^{+}$upon irradiation. It also remains to be seen how p62-mediated aggregation of caspase-8 activates downstream executioner caspases. One possibility is that p62associated caspases- $8^{+}$aggresomes prolong the half-life of polyubiquitinated caspase- 8 beyond the necessary threshold for the activation of downstream executioner caspases, instead of being targeted to the proteasome, as previously suggested [25]. Taken together, our study provides molecular insights into the functional interplay between autophagy and programmed cell death pathways regulated by p62. We, thus, propose that $p 62$ is a druggable target for the manipulation of sensitivity to death stimuli in cancer cells, typically resistant to both intrinsic and extrinsic cell death stimuli (Supplementary Fig. 4). In addition to cancer therapeutics, our findings may have broad biomedical applications since imbalance between autophagy and apoptosis are often involved in many human diseases. 


\section{DATA AVAILABILITY}

All data is available in the manuscript file and its supplementary information file or available upon request.

\section{REFERENCES}

1. Gump JM, Thorburn A. Autophagy and apoptosis: what is the connection? Trends Cell Biol. 2011;21:387-92.

2. Cecconi $F$, Levine B. The role of autophagy in mammalian development: cell makeover rather than cell death. Dev Cell. 2008;15:344-57.

3. Tuzlak S, Kaufmann T, Villunger A. Interrogating the relevance of mitochondrial apoptosis for vertebrate development and postnatal tissue homeostasis. Genes Dev. 2016;30:2133-51

4. Thorburn A. Apoptosis and autophagy: regulatory connections between two supposedly different processes. Apoptosis. 2008;13:1-9.

5. Singh R, Letai A, Sarosiek K. Regulation of apoptosis in health and disease: the balancing act of BCL-2 family proteins. Nat Rev Mol Cell Biol. 2019;20:175-93.

6. Mizushima N, Komatsu M. Autophagy: renovation of cells and tissues. Cell. 2011;147:728-41.

7. Kung CP, Budina A, Balaburski G, Bergenstock MK, Murphy M. Autophagy in tumor suppression and cancer therapy. Crit Rev Eukaryot Gene Expr. 2011;21:71-100.

8. Mathew R, Karantza-Wadsworth V, White E. Role of autophagy in cancer. Nat Rev Cancer. 2007;7:961-7.

9. Thorburn A, Thamm DH, Gustafson DL. Autophagy and cancer therapy. Mo Pharm. 2014:85:830-8.

10. Wu H, Che X, Zheng Q, Wu A, Pan K, Shao A, et al. Caspases: a molecular switch node in the crosstalk between autophagy and apoptosis. Int J Biol Sci. 2014;10:1072-83.

11. Pattingre S, Tassa A, Qu X, Garuti R, Liang XH, Mizushima N, et al. Bcl-2 antiapoptotic proteins inhibit Beclin 1-dependent autophagy. Cell. 2005;122:927-39.

12. Rubinsztein DC, Codogno $P$, Levine B. Autophagy modulation as a potential therapeutic target for diverse diseases. Nat Rev Drug Discov. 2012;11:709-30.

13. Mulcahy Levy JM, Thorburn A. Autophagy in cancer: moving from understanding mechanism to improving therapy responses in patients. Cell Death Differ. 2020;27:843-57.

14. Xu R, Ji Z, Xu C, Zhu J. The clinical value of using chloroquine or hydroxychloroquine as autophagy inhibitors in the treatment of cancers: a systematic review and meta-analysis. Medicine. 2018;97:e12912.

15. Bachmair A, Finley D, Varshavsky A. In vivo half-life of a protein is a function of its amino-terminal residue. Science. 1986;234:179-86.

16. Tasaki T, Sriram SM, Park KS, Kwon YT. The N-end rule pathway. Annu Rev Biochem. 2012;81:261-89.

17. Kwon YT, Kashina AS, Davydov IV, Hu RG, An JY, Seo JW, et al. An essential role of $\mathrm{N}$-terminal arginylation in cardiovascular development. Science. 2002;297:96-99.

18. Sriram SM, Kwon YT. The molecular principles of $\mathrm{N}$-end rule recognition. Nat Struct Mol Biol. 2010;17:1164-5.

19. Kwon YT, Xia Z, Davydov IV, Lecker SH, Varshavsky A. Construction and analysis of mouse strains lacking the ubiquitin ligase UBR1 (E3alpha) of the $\mathrm{N}$-end rule pathway. Mol Cell Biol. 2001;21:8007-21.

20. Tasaki T, Kwon YT. The mammalian $\mathrm{N}$-end rule pathway: new insights into its components and physiological roles. Trends Biochem Sci. 2007;32:520-8.

21. Cha-Molstad H, Sung KS, Hwang J, Kim KA, Yu JE, Yoo YD, et al. Amino-terminal arginylation targets endoplasmic reticulum chaperone BiP for autophagy through p62 binding. Nat Cell Biol. 2015;17:917-29.

22. Cha-Molstad H, Yu JE, Feng Z, Lee SH, Kim JG, Yang P, et al. p62/SQSTM1/ Sequestosome-1 is an N-recognin of the $\mathrm{N}$-end rule pathway which modulates autophagosome biogenesis. Nat Commun. 2017;8:102.

23. Cha-Molstad H, Lee SH, Kim JG, Sung KW, Hwang J, Shim SM, et al. Regulation of autophagic proteolysis by the $\mathrm{N}$-recognin SQSTM1/p62 of the $\mathrm{N}$-end rule pathway. Autophagy. 2018;14:359-61.

24. Ullman E, Pan JA, Zong WX. Squamous cell carcinoma antigen 1 promotes caspase-8-mediated apoptosis in response to endoplasmic reticulum stress while inhibiting necrosis induced by lysosomal injury. Mol Cell Biol. 2011;31:2902-19.

25. Jin Z, Li Y, Pitti R, Lawrence D, Pham VC, Lill JR, et al. Cullin3-based polyubiquitination and p62-dependent aggregation of caspase-8 mediate extrinsic apoptosis signaling. Cell. 2009;137:721-35.

26. Attner P, Du J, Nasman A, Hammarstedt L, Ramqvist T, Lindholm J, et al. The role of human papillomavirus in the increased incidence of base of tongue cancer. Int J Cancer. 2010;126:2879-84.

27. Chaturvedi AK, Engels EA, Anderson WF, Gillison ML. Incidence trends for human papillomavirus-related and -unrelated oral squamous cell carcinomas in the United States. J Clin Oncol. 2008:26:612-9.

28. Hammarstedt L, Lindquist D, Dahlstrand H, Romanitan M, Dahlgren LO, Joneberg $\mathrm{J}$, et al. Human papillomavirus as a risk factor for the increase in incidence of tonsillar cancer. Int J Cancer. 2006;119:2620-3.
29. Kobayashi K, Hisamatsu K, Suzui N, Hara A, Tomita H, Miyazaki T. A review of HPVrelated head and neck cancer. J Clin Med. 2018;7:241.

30. Gillison ML, Chaturvedi AK, Anderson WF, Fakhry C. Epidemiology of human papillomavirus-positive head and neck squamous cell carcinoma. J Clin Oncol. 2015;33:3235-42.

31. Jethwa AR, Khariwala SS. Tobacco-related carcinogenesis in head and neck cancer. Cancer Metastasis Rev. 2017;36:411-23.

32. Ang MK, Patel MR, Yin XY, Sundaram S, Fritchie $K$, Zhao N, et al. High XRCC1 protein expression is associated with poorer survival in patients with head and neck squamous cell carcinoma. Clin Cancer Res. 2011;17:6542-52.

33. Fakhry C, Westra WH, Li S, Cmelak A, Ridge JA, Pinto H, et al. Improved survival of patients with human papillomavirus-positive head and neck squamous cell carcinoma in a prospective clinical trial. J Natl Cancer Inst. 2008;100:261-9.

34. Hong AM, Dobbins TA, Lee CS, Jones D, Harnett GB, Armstrong BK, et al. Human papillomavirus predicts outcome in oropharyngeal cancer in patients treated primarily with surgery or radiation therapy. Br J Cancer. 2010;103:1510-7.

35. Sethi S, Ali-Fehmi R, Franceschi S, Struijk L, van Doorn LJ, Quint W, et al. Characteristics and survival of head and neck cancer by HPV status: a cancer registrybased study. Int J Cancer. 2012;131:1179-86.

36. Lill C, Kornek G, Bachtiary B, Selzer E, Schopper C, Mittlboeck M, et al. Survival of patients with HPV-positive oropharyngeal cancer after radiochemotherapy is significantly enhanced. Wien Klin Wochenschr. 2011;123:215-21.

37. Mellin H, Friesland S, Lewensohn R, Dalianis T, Munck-Wikland E. Human papillomavirus (HPV) DNA in tonsillar cancer: clinical correlates, risk of relapse, and survival. Int J Cancer. 2000;89:300-4.

38. Sedaghat AR, Zhang Z, Begum S, Palermo R, Best S, Ulmer KM, et al. Prognostic significance of human papillomavirus in oropharyngeal squamous cell carcinomas. Laryngoscope. 2009;119:1542-9.

39. Worden FP, Kumar B, Lee JS, Wolf GT, Cordell KG, Taylor JM, et al. Chemoselection as a strategy for organ preservation in advanced oropharynx cancer: response and survival positively associated with HPV16 copy number. J Clin Oncol. 2008;26:3138-46.

40. Jung YS, Najy AJ, Huang W, Sethi S, Snyder M, Sakr W, et al. HPV-associated differential regulation of tumor metabolism in oropharyngeal head and neck cancer. Oncotarget. 2017;8:51530-41.

41. Cho WJ, Kessel D, Rakowski J, Loughery B, Najy AJ, Pham T, et al. Photodynamic therapy as a potent radiosensitizer in head and neck squamous cell carcinoma. Cancers. 2021;13:1193.

42. Stolz A, Ernst A, Dikic I. Cargo recognition and trafficking in selective autophagy. Nat Cell Biol. 2014;16:495-501.

43. Liu JL, Chen FF, Lung J, Lo CH, Lee FH, Lu YC, et al. Prognostic significance of p62/ SQSTM1 subcellular localization and LC3B in oral squamous cell carcinoma. $\mathrm{Br} J$ Cancer. 2014;111:944-54.

44. Wang $\mathrm{Y}$, Zhang N, Zhang L, Li R, Fu W, Ma K, et al. Autophagy regulates chromatin ubiquitination in DNA damage response through elimination of SQSTM1/p62. Mol Cell. 2016;63:34-48.

45. Peter ME, Krammer PH. The CD95(APO-1/Fas) DISC and beyond. Cell Death Differ. 2003;10:26-35.

46. Ashkenazi A, Dixit VM. Death receptors: signaling and modulation. Science. 1998;281:1305-8

47. Gonzalvez F, Lawrence D, Yang B, Yee S, Pitti R, Marsters $S$, et al. TRAF2 sets a threshold for extrinsic apoptosis by tagging caspase- 8 with a ubiquitin shutoff timer. Mol Cell. 2012;48:888-99.

\section{ACKNOWLEDGEMENTS}

We thank the members of the Kim and Kwon laboratories for their critical discussions.

\section{AUTHOR CONTRIBUTIONS}

S.H.L., W.J.C, Y.T.K., and H-R.C.K. designed the experiments, interpreted the results, and prepared the manuscript. S.H.L. and W.J.C performed the majority of the experiments. A.J.N., A-D.S., T.P., and W.S. contributed to p62 immunohistochemistry and A.J.N., A-D.S., and T.P. performed proximity ligation assay as well as the interpretation of the results. W.J.C., J.R., B.L., W.K.C., and H.E.K. contributed to ionizing radiation treatment (XRT). C.H.J. performed immunoprecipitation assays and interpreted the results. H-R.C.K., Y.T.K., W.S., and H.E.K. provided guidance, specialized reagents, and expertise.

\section{FUNDING}

This research was supported by NIH grants CA 123362 (H-R.C.K.) and DE023181 (IK, H-R.C.K and HEK), Korean National Science Foundation Brain Pool Program Award (HR.C.K.), the International Research \& Development Program of the National Research 
10

Foundation of Korea (NRF) funded by the Ministry of Science and ICT (2019K1A3A1A12070180 to S.H.L.), NRF grant funded by the Korea government (MSIT) (No. NRF-2020R1A5A1019023 to Y.T.K.), and Basic Science Research Program through NRF funded by the Ministry of Education (NRF-2021R1A2B5B03002614 to Y. T.K.), and SNU Hospital (to Y.T.K.).

\section{ETHICS APPROVAL}

Collection and utilization of tissue specimens were approved by the Institutional Review Board (IRB) of Wayne State University.

\section{COMPETING INTERESTS}

The authors declare no competing interests.

\section{ADDITIONAL INFORMATION}

Supplementary information The online version contains supplementary material available at https://doi.org/10.1038/s41419-021-04301-7.

Correspondence and requests for materials should be addressed to Yong Tae Kwon or Hyeong-Reh C. Kim.
Reprints and permission information is available at http://www.nature.com/ reprints

Publisher's note Springer Nature remains neutral with regard to jurisdictional claims in published maps and institutional affiliations.

cc) (i)

Open Access This article is licensed under a Creative Commons Attribution 4.0 International License, which permits use, sharing, adaptation, distribution and reproduction in any medium or format, as long as you give appropriate credit to the original author(s) and the source, provide a link to the Creative Commons license, and indicate if changes were made. The images or other third party material in this article are included in the article's Creative Commons license, unless indicated otherwise in a credit line to the material. If material is not included in the article's Creative Commons license and your intended use is not permitted by statutory regulation or exceeds the permitted use, you will need to obtain permission directly from the copyright holder. To view a copy of this license, visit http://creativecommons. org/licenses/by/4.0/.

(c) The Author(s) 2021 